\title{
Bispectral Index Monitoring in a Severe Comatose Patient During Surgery
}

\author{
Susana Fernández Galinski ${ }^{*}, 1$, Enriqueta Barrera ${ }^{1}$, Antonio Valls ${ }^{2}$, Milagros Montero ${ }^{3}$, \\ Juan Carlos Alvarez ${ }^{1}$ and Fernando Escolano ${ }^{1}$
}

\author{
${ }^{1}$ Anaesthesiology Department, ${ }^{2}$ Neurophysiology Department, ${ }^{3}$ Internal Medicine Department, Hospital Universitario \\ del Mar, Universidad Autónoma de Barcelona, Spain
}

\begin{abstract}
A comatose patient required a tracheostomy. His Glasgow Coma Score was 4 and he was intubated with mydriatic and isochoric pupils, without corneal and light reflexes. Two previous EEGs showed signs of severe and diffuse encephalopathy (arreactive delta and theta rhythms) and there were no somatosensory evoked potentials recordable at the scalp. When the tracheostomy procedure was carried out, the Bispectral Index (BIS) helped to titrate the hypnotic and analgesic requirements keeping the haemodynamic parameters stable.
\end{abstract}

\section{IMPLICATIONS STATEMENT}

A comatose patient had to undergo surgery. We guided the hypnotic and analgesic requirements by the bispectral index.

The bispectral index (BIS) has been used in severely comatose patients as an assessment of the onset of brain death [1]. Moreover, in patients in a persistent vegetative state, BIS has helped to guide anaesthetic depth during surgery [2]. We show the clinical state, electrophysiological tests and the anaesthetic management of a patient in coma after cardiac arrest due to near drowning, who needed to undergo a tracheostomy.

\section{CASE REPORT}

A 72 yr old $80 \mathrm{~kg}$ male, was resuscitated (advanced CPR lasting 40 minutes). He was endotracheal intubated with a Glasgow Coma Score (GCS) of 3, his pupils were mydriatic and isochoric, without corneal or light reflexes. At the intensive care unit (ICU) and for two weeks, he went on ventilated using pressure support mode, because he had spontaneous breathing. The neurological condition evolved to a GCS equal to $4+$ tube, with a rigid posture, sporadic passive eye opening and weak withdrawal responses to the nociceptive stimulus. Two EEG were carried out, the first, $24 \mathrm{~h}$ after admission to ICU and the second a week later, both showed signs of severe and diffuse encephalopathy (arreactive delta and theta rhythms). The somatosensory evoked potentials recorded at the scalp, were absent when the posterior tibial nerves were stimulated.

After two weeks, it was decided to perform a tracheostomy. At the operating theatre, the conventional monitoring went on while the BIS was measured with an Aspect A-2000 monitor / $\mathrm{BIS}_{\mathrm{TM}}$ quatro sensor (Aspect Medical Systems Inc., Newton MA). We guided the hypnotic and analgesic requirements by BIS, observing the haemodynamic parame-

*Address correspondence to this author at the Department of Anaesthesiology, Hospital Universitario del Mar, UAB, Paseo Marítimo 25-29 08003 Barcelona, Spain; Tel: 34932483350; Fax: 34932483617;

E-mail: 18569@imas.imim.es ters, too. At the beginning, the systolic blood pressure (SBP) was higher than $200 \mathrm{mmHg}$ while BIS values ranged from 90 to 100 , so we used intravenous labetalol $(5 \mathrm{mg})$ and administered cis atracurium $(4 \mathrm{mg})$, to adapt the mechanical ventilation and to attenuate the rigidity (EMG activity 50 ). Thus, the SBP decreased to $150 \mathrm{mmHg}$ while the BIS and EMG values fell to almost 50 and 30 respectively. As BIS and EMG values were restored, we began to administer sevoflurane at $1,5 \%$ end -tidal concentration, a dose that allowed him to reach an anaesthesia depth ready for surgery (BIS 60). A few minutes later, while BIS was around 60, the sevoflurane had to be decreased to $1 \%$ and ephedrine had to be administered because the SBP had dropped to $70 \mathrm{mmHg}$. The extension of the neck, provoked a new abrupt increase of BIS value with low EMG activity, so we added a remifentanil infusion at a rate of 0.1 ug. $\mathrm{kg}^{-1} \cdot \mathrm{min}^{-1}$, which caused a deep fall of the BIS value to 28 with a suppression ratio (SR) of $47 \%$ while the SBP was around $110 \mathrm{mmHg}$. The surgical stimulation, abruptly raised the BIS value to 60 but the SBP continued near to $110 \mathrm{mmHg}$. A few minutes later, the introduction of the tracheal cannula provoked coughing and movement, increasing the BIS value to 90 (EMG activity 50) without any change in SBP. We increased the sevoflurane concentration $(1,5 \%)$ and the rate of remifentanil infusion to $0.3 \mathrm{ug} \cdot \mathrm{kg}^{-1} \cdot \mathrm{min}^{-1}$ while cis atracurium was also administered. This manoeuvre led to a new drop of the BIS value to 20 (SR $35-61 \%)$. Five minutes later, a brief period of increased $\mathrm{SBP}(170 \mathrm{mmHg})$ was observed. From this time to the end of surgery, as the noxious stimulation was diminishing, we decreased the sevoflurane and remifentanil doses, observing a slope of the BIS trend towards 60 together with a decrease in SBP, then remifentanil and sevoflurane were stopped (Fig. 1). The raw EEG trace obtained at this moment showed a low- voltage beta pattern alternating with waves of higher amplitude which could correspond to the ECG signal.

\section{DISCUSSION}

The bispectral index was developed to monitor the depth of unconsciousness but the usefulness of it in brain damaged patients is being investigated. Thus, BIS monitoring has been used in critically ill children to guide the barbiturate coma [3]. On the other hand, it has detected the ischaemic - in- 


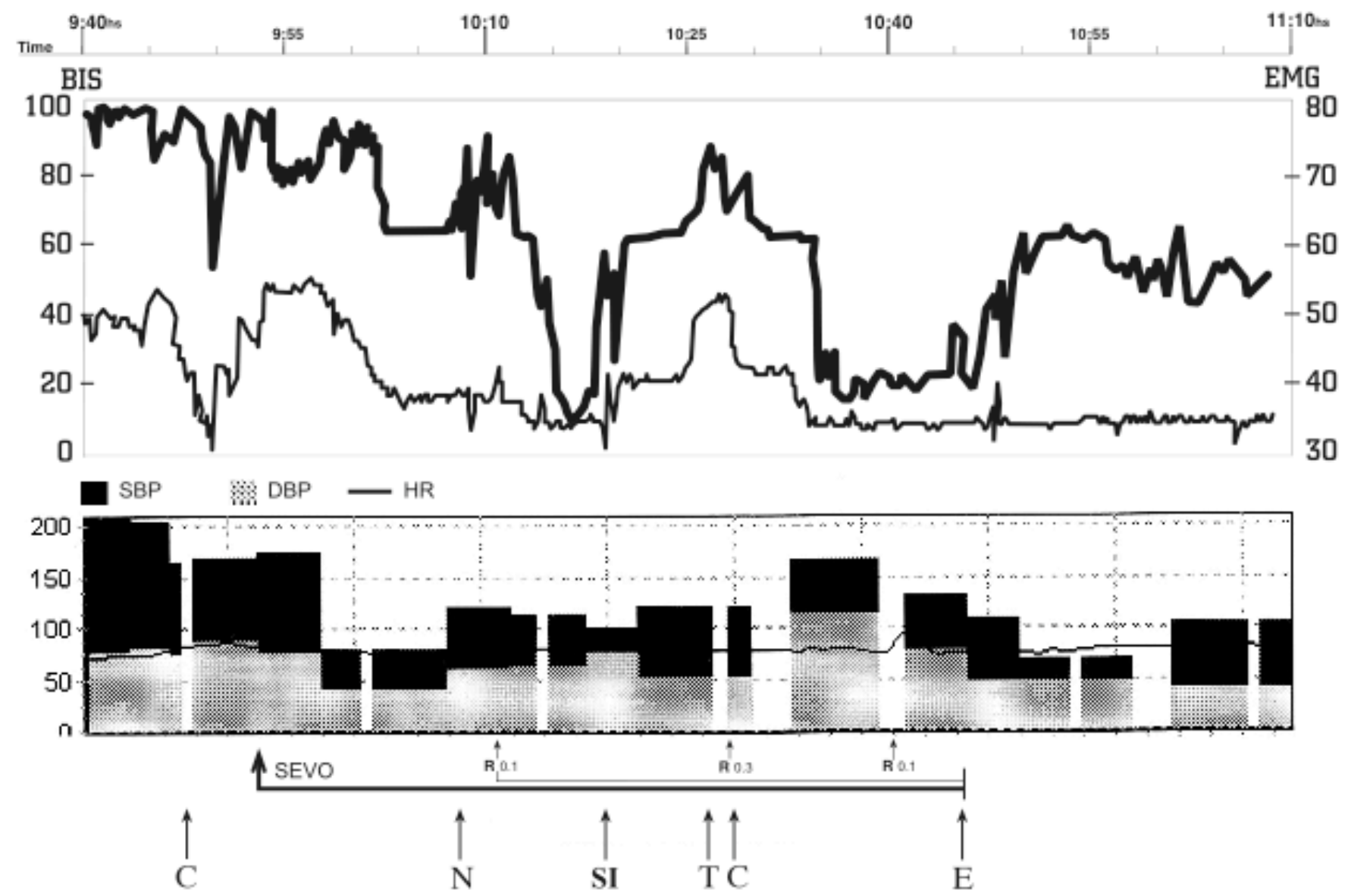

Fig. (1). Top panel: BIS and EMG trends during surgery. Bottom panel: Recording of non-invasive systemic blood pressure. SBP: systolic blood pressure; DBP: diastolic blood pressure; HR: heart rate. C: cis- atracurium; SEVO: sevoflurane; N: extension of the neck; R: remifentanil (ug.kg $\left.{ }^{-1} \cdot \mathrm{min}^{-1}\right)$; SI: skin incision; T: tracheal cannula; E: stop remifentanil and sevoflurane.

duced cortical dysfunction at the time of aortic cross clamping during cardiac surgery [4], moreover, the BIS monitor can show brain death onset [1].

Several reports have confirmed that changes in BIS index correlate with haemodynamic variables, mainly with mean arterial pressure $[5,6]$. In this patient, we decided to guide the anaesthesia by BIS monitoring because he could have autonomic dysregulation as a result of his anoxic injury, then the variations of the haemodynamic parameters would not truly reflect his analgesic and hypnotic requirements [7]. In fact, this management contributed to the haemodynamic stability of the patient.

On the other hand, a significant correlation between BIS values and GCS scores has been demonstrated in patients with head injury, although a high degree of scatter of BIS values for any GCS score was also found. In addition, an empiric BIS cut-off value of 50 was proposed to differentiate unconscious patients (coma, persistent vegetative state) from conscious (minimally conscious state) patients [8-10]. In our patient, the high baseline BIS value was neither in accordance with his GCS of 4, nor with the established cut-off value since our patient was in coma with a BIS between 90100 and clearly it could not be indicating consciousness. After ruling out technical interferences because we had a good signal quality index, we believed that an increased ac- tivity of the EMG could be disguising the BIS value, but although the neuromuscular blockade was useful to adapt the mechanical ventilation, it had a very short effect on the BIS value [11]. It was the administration of sevoflurane that decreased BIS and SBP values as happens in any general anaesthesia. Therefore, we think (although we can not affirm due to the lack of a raw EEG trace) that our patient could have been in alpha coma, a state in which the EEG resembles that found during normal wakefulness, consisting predominantly of activity in the alpha frequency range. This is a state that indicates severe disturbance in thalamo -cortical physiology [12].

On the other hand, although the BIS index is mainly an indicator of hypnosis, it is well established that the level of analgesia can modify hypnosis. The fact that BIS values changed in the presence of different intensities of noxious stimuli, could be taken as an indirect indicator of the degree of analgesia [13]. Thus, while $1 \%$ sevoflurane was maintained, remifentanil blunted the abrupt increase of BIS values during the extension of the neck, and it prevented the BIS value exceeding 60 during the surgical incision, which is a stronger nociceptive stimulus.

In this case, the high baseline BIS value did not reflect the unconscious state of the patient probably due to an alpha coma state. The BIS variations observed during anaesthetic 
induction and surgery were similar to a patient with normal cerebral function.

This was not a conventional anaesthetic management, but it allowed us to adapt the anaesthetic drugs to the true requirements of the patient maintaining his haemodynamic parameters stable

\section{REFERENCES}

[1] Vivien B, Paqueron X, Le Cosquer P, Langeron O, Coriat P, Riou B. Detection of brain death onset using the bispectral index in severely comatose patients. Intensive Care Med 2002; 28: 419-25.

[2] Pandit JJ, Schmelzle- Lubiecki B, Goodwin M, Saeed N. Bispectral index- guide management of anaesthesia in permanent vegetative state. Anaesthesia 2002; 57: 1190-4.

[3] Prins SA, de Hoog M, Blok JH, Tibboel D, Visser GH. Continuous non-invasive monitoring of barbiturate coma in critically ill children using the BispectralO (BISO) Index Monitor. Crit Care 2007; 11: R108.

[4] Ellenberger C, Diaper J, Licker M, Panos A. Bispectral index and detection of acute brain injury during cardiac surgery. Eur J Anaesthesiol 2007; 24: 807-9.

[5] Guignard B, Menigaux C, Dupont X, Fletcher D, Chauvin M. The effect of remifentanil on the bispectral index change and haemody- namic responses after orotracheal intubation. Anesth Analg 2000; 90: 161-7.

[6] Casati L, Fernández Galinski S, Barrera E, Pol O, Puig MM. Isoflurane requirements during combined general-epidural anestesia for major abdominal surgery. Anesth Analg 2002; 94: 1331-7.

[7] Diesing TS, Wijdicks EF. Arc de cercle and dysautonomia from anoxic injury. Mov Disord 2006; 21: 868-9.

[8] Paul DB, Umamaheswara Rao GS. Correlation of Bispectral Index with Glasgow Coma Score in mild and moderate head injuries. J Clin Monit Comput 2006; 20: 399-404.

[9] Schnakers C, Majerus S, Laureys S. Bispectral analysis of electroencephalogram signals during recovering from coma: preliminary findings. Neuropsychol Rehabil 2005; 15: 381-8.

[10] Deogaonkar A, Gupta R, DeGeorgia M, et al. Bispectral index monitoring correlates with sedation scales in brain-injured patients Crit Care Med 2004; 32: 2403-6.

[11] Liu, N, Chazot T, Fischler M. Changes of bispectral index after a bolus dose of muscle relaxant. Anesth Analg 2006; 103: 777.

[12] Fung PC, Tucker RP. Alpha rhythm and alpha-like activity in coma. Clin Electroencephalogr 1984; 15: 167-72.

[13] Fernández Galinski S, Bermejo S, Mansilla R, Pol O, Puig MM. Comparative assessment of the effects of alfentanil, esmolol, or clonidine when used as adjuvants during induction of general anaesthesia. Eur J Anaesthesiol 2004; 21: 476-82. 